\title{
Characteristics of the gut microbiome in patients with prediabetes and type 2 diabetes
}

\author{
Zewen Zhang ${ }^{\text {Equal first author, } 1}$, Tian Tian ${ }^{\text {Equal first author, } 1}{ }^{1}$, Zhen Chen ${ }^{1}$, Lirong Liu ${ }^{1}$, Tao Luo ${ }^{1}$, Dai Jianghong ${ }^{\text {Corresp. } 1}$ \\ ${ }^{1}$ School of Public Health, Xinjiang Medical University, Urumqi, China \\ Corresponding Author: Dai Jianghong \\ Email address: epidjh@163.com
}

Background. Gut microbiome has recently been identified as a new potential risk factor in addition to well-known diabetes risk factors. The aim of this study was to analyze the differences in the composition of gut microbiome in prediabetes(PreDM), type 2 diabetes mellitus (T2DM) and non-diabetic controls.

Methods. 180 participants were recruited for this study: 60 with T2DM, 60 with PreDM and 60 nondiabetics (control group). Fecal samples were collected from the participants and genomic DNA was extracted. The Composition and diversity of gut microbiome were investigated in fecal DNA samples using Illumina sequencing of the V3 V4 regions of 16sRNA.

Results. There were significant differences in the number of bacteria among patients with PreDM and T2DM and the control group. Compared with the control group, Proteobacteria bacteria were significantly higher in the PreDM group $(P=0.006)$. On the genus level, Compared with the control group, the relative abundance of Prevotella and Alloprevotella was significantly higher in the T2DM group $(P=0.016$, $P=0.018$ ), and the relative abundance of Paraprevotella in T2DM and PreDM groups was lower than that in the control group $(P=0.011, P=0.045)$. Compared with the PreDM group and the control group, the relative abundance of Bacteroides in the T2DM group was significantly lower $(P=0.019, P=0.002)$.

Conclusions. The present study found significant differences in the gut microbiome between PreDM, T2DM and non-diabetic individuals, specifically at the genus level, suggesting that early intervention in PreDM patients could have implications for gut flora transitioning to T2DM. In addition, these results may be valuable for developing strategies to control T2DM by modifying the gut microbiome. 
1 Characteristics of the gut microbiome in patients with

2 prediabetes and type 2 diabetes

3 Zewen Zhang ${ }^{1, *}$, Tian Tian ${ }^{1, *}$, Zhen Chen $^{1}$, Lirong Liu ${ }^{1}$, Tao Luo ${ }^{1}$, Jianghong Dai ${ }^{1}$

$4 \quad{ }^{1}$ Department of Epidemiology and Biostatistics, School of Public Health, Xinjiang Medical

5 University, Urumqi, the Xinjiang Uygur Autonomous Region, China

$6 \quad{ }^{*}$ These authors contributed equally

7 Corresponding Author:

8 Jianghong Dai ${ }^{1}$

9 No.393 Xinyi Road, Xinshi District, Urumqi, the Xinjiang Uygur Autonomous Region, 830011,

10 China

11 Email address: epidjh@163.com

\section{Abstract}

Background Gut microbiome has recently been identified as a new potential risk factor in addition to well-known diabetes risk factors. The aim of this study was to analyze the differences in the composition of gut microbiome in prediabetes(PreDM), type 2 diabetes mellitus (T2DM) and non-diabetic controls.

Methods 180 participants were recruited for this study: 60 with T2DM, 60 with PreDM and 60 non-diabetics (control group). Fecal samples were collected from the participants and genomic DNA was extracted. The Composition and diversity of gut microbiome were investigated in fecal DNA samples using Illumina sequencing of the V3 V4 regions of 16sRNA.

Results There were significant differences in the number of bacteria among patients with PreDM and T2DM and the control group. Compared with the control group, Proteobacteria bacteria were significantly higher in the PreDM group $(P=0.006)$. On the genus level, Compared with the control group, the relative abundance of Prevotella and Alloprevotella was significantly higher in the T2DM group $(P=0.016, P=0.018)$, and the relative abundance of Paraprevotella in T2DM and PreDM groups was lower than that in the control group $(P=0.011, P=0.045)$. Compared with the PreDM group and the control group, the relative abundance of Bacteroides in the T2DM group was significantly lower $(P=0.019, P=0.002)$. Conclusions The present study found significant differences in the gut microbiome between PreDM, T2DM and non-diabetic individuals, specifically at the genus level, suggesting that early intervention in PreDM patients could have implications for gut flora transitioning to T2DM. In addition, these results may be valuable for developing strategies to control T2DM by modifying the gut microbiome. 
Keywords: Gut Microbiome; Type 2 Diabetes mellitus; Prediabetes.

\section{Introduction}

Type 2 diabetes mellitus (T2DM) is a metabolic syndrome characterized by insulin dysfunction and abnormal glucose and lipid metabolism that has become one of the world's most common public health problems(Gaike et al. 2020). According to the statistics released by the International Diabetes Federation(Cho et al. 2018), in 2015 the global number of people aged 2079 years with diabetes was 415 million, a number that will rise to 642 million by 2040 . The standardized prevalence of diagnosed and undiagnosed diabetes in the Chinese adult population was estimated to be $10.9 \%$ in 2013(Wang et al. 2017). Prediabetes(PreDM) is defined as a condition in which blood glucose levels are higher than normal, but below the threshold for the diagnosis of diabetes(Allin et al. 2018). Individuals with PreDM often present overweight, with insulin resistance, and low levels of inflammation, and they suffer from an increased risk of T2DM and ischemic cardiovascular disease. It is estimated(Wang et al. 2017) that the prevalence of PreDM in China was $35.7 \%$ in 2013, and without intervention in the prediabetic population $70 \%$ will eventually progress to diabetes mellitus, with an annual conversion rate of $5 \%$ to $10 \%$ (Tabak et al. 2012). It is important to intervene proactively in the prediabetic population to interrupt or slow down the progression to T2DM. The gut microbiome has been shown to be an important factor in the development of T2DM, along with genetic, environmental, dietary, and behavioral lifestyle factors(Gaike et al. 2020; Ma et al. 2019).

The gut is the largest immune organ in the human body, and the intestinal flora residing in the gut plays a role in maintaining intestinal homeostasis, metabolism and immunity. It is also known as the "second genome"(Lynch \& Pedersen 2016). Healthy adult gut microbiota are dominated by Bacteroidetes and Firmicutes ( $>90 \%$ ) but also include smaller proportions of Actinobacteria, Proteobacteria, Fusobacteria and Verrucomicrobia(Hollister et al. 2014; Naseer et al. 2014). 
62

63

64

The gut microbiome plays an important role in regulating energy metabolism and inflammation, and is closely related to a variety of chronic diseases, such as obesity, T2DM, inflammatory bowel disease, and rheumatoid arthritis(Festi et al. 2014; Komaroff 2017; Lynch \& Pedersen 2016; Zhang et al. 2015). Some studies have indicated that gut microorganisms directly increase intestinal uptake of monosaccharides and promote hepatic production of triglycerides associated with insulin resistance(Larsen et al. 2010b). It has been suggested(Sedighi et al. 2017) that the gut microbiome can increase energy absorption from food, cause chronic low-grade inflammation, regulate fatty acid metabolism, secrete derived peptides and increase the production of metabolic endotoxins (lipopolysaccharides), leading to a chronic low rate of inflammation and insulin resistance. Previous studies have demonstrated that the quantity of Firmicutes, Bifidobacteria and Clostridia was significantly lower in patients with T2DM compared with that in healthy individuals(Karlsson et al. 2013; Sato et al. 2014), whereas the number of Bacteroidetes and beta Proteobacteria was significantly higher(Qiu et al. 2019). It was shown that the Bacteroidetes/Firmicutes ratio in T2DM was positively and significantly correlated with plasma glucose concentration, but appeared to be independent of body weight, confirming that it was associated with reduced glucose tolerance.

Although the causal relationship between dysbiosis of the gut microbiome and T2DM is not clear, changes in the intestinal microbiome of patients with T2DM have been confirmed(Lambeth et al. 2015; Sedighi et al. 2017). There are many reports on the intestinal microbiome and T2DM, but the results of the studies differ. T2DM treatment drugs may be one of the influencing factors. Studies have shown that diabetes treatment drugs (such as metformin) may confuse the relationship between intestinal flora dysbiosis and T2DM(Forslund et al. 2015). In addition, it is not known whether there is any change in the gut microbiome of prediabetic patients and how it differs from that of T2DM and non-diabetic individuals. Few previous(Gaike et al. 2020; Zhong et al. 2019) studies have analyzed three groups of people: those with newly diagnosed T2DM, PreDM and non-diabetes. Therefore, this study recruited patients with newly diagnosed diabetes and PreDM to elucidate the characteristics of the intestinal microbiota in 
89

90

91

92

93

94

95

96

97

98

99

100

101

102

103

104

105

106

107

108

109

110

111

112

113

114

115

patients with preDM and T2DM.

\section{Materials \& Methods}

\section{Study population}

The study was approved by Ethics Committee of the First Affiliated Hospital of Xinjiang Medical University(20191113-05).60 patients with newly diagnosed T2DM, 60 with preDM from the first Affiliated Hospital of Xinjiang Medical University and 60 healthy participants from the Health Management Hospital of Xinjiang Medical University were recruited for this study and signed informed consent. All participants were between 20- and 65-years-old. Healthy participants were defined as having fasting plasma glucose $<5.6 \mathrm{mmol} / \mathrm{L}$. Participants with T2DM were required to meet the following inclusion criteria: (i) fasting blood glucose test (FBG) $\geq 7 \mathrm{mmol} / \mathrm{L}$ and/or 2-h fasting oral glucose tolerance test (OGTT) $\geq 11.1 \mathrm{mmol} / \mathrm{L}$; and (ii) all cases of T2DM were newly diagnosed. PreDM was defined as FBG of $6.1-7.0 \mathrm{mmol} / \mathrm{L}$ or $\mathrm{HbA}_{1 \mathrm{c}}$ levels of $6.0 \%-6.5 \%$. To eliminate the effects of other factors on the gut microbiota, we excluded individuals according to certain criteria: (i) age less than 20 or greater than 60 years; (ii) antibiotic usage within 2 months; (iii) habitual probiotic use; and (iv) acute and chronic gastrointestinal diseases. Information regarding demographics, diet, alcohol and tobacco use was obtained by means of a survey questionnaire. Dietary habits were assessed using a validated Food Frequency Questionnaire (FFQ). All stool samples were collected using sterile cups instantly after defecation, and sent to the laboratory within 1 hour using Styrofoam containers containing ice packs, and immediately stored in freezer at $-80^{\circ} \mathrm{C}$.

\section{DNA extraction, PCR amplification, and 16S rRNA gene sequencing} Total DNA of microorganisms was extracted from all 180 samples using the FastDNA Spin Kit for Soil from mp biomedicals. The procedure was performed according to the instructions. DNA was quantified using a NanoDrop ND-1000 spectrophotometer (Rockland Company, USA) and stored at $-80^{\circ} \mathrm{C}$ for later use. The extracted genomic DNA was used to construct an amplicon 
116 library by amplifying the V3 V4 region of the $16 \mathrm{~S}$ rRNA gene. PCR was performed using the

117 following conditions: initial denaturation at $95^{\circ} \mathrm{C}$ for $3 \mathrm{~min} ; 25$ cycles with 1 cycle consisting of

$11895^{\circ} \mathrm{C}$ for $30 \mathrm{~s}, 55^{\circ} \mathrm{C}$ for $30 \mathrm{~s}$, and $72^{\circ} \mathrm{C}$ for $30 \mathrm{~s}$; and a final extension step at $72^{\circ} \mathrm{C}$ for $7 \mathrm{~min}$.

119 After the reaction, all reaction products were detected by $1.5 \%$ agar gel electrophoresis (ethidium

120 bromide staining) to detect the amplified fragment size. A QIAquick GelExtraction Kit

121 (QIAGEN, Germany) was used to recover and purify the target band adhesive. An Illumina

122 Miseq high-throughput sequencing platform (Illumina) was used to sequence the PCR products

123 in the $16 \mathrm{~S}$ RNA V3 V4 region.

124

125

126

127

128

129

130

131

132

133

134

135

\section{Statistical analysis}

Statistical analysis was performed using SPSS 21.0 software and R 3.43. Quality control and filtering of the raw sequencing data in order to obtain high quality sequencing data and improve the accuracy of subsequent analyses(Supplemental files1). To analyze the differences among the groups, normally distributed variables were assessed with one-way analysis of variance (ANOVA) followed by the LSD test or Dunnett's test; categorical variables were assessed with the $\chi^{2}$ test. Differences in dietary frequencies among the three groups were tested using the $\chi^{2}$ test, and if the differences were significant, Z-test was used for pair comparison and the Bonferroni method was used to adjust for $\mathrm{P}$ values. The relative abundances were compared across the three groups using Kruskal-Wallis rank sum tests followed, if significant, by pair-wise comparison, and false discovery rate (FDR) using the Benjamini-Hochberg method was applied to correct the significant p-values. Alpha diversity was assessed using the Observed Species index, Chao1 index, ACE index, Shannon index, Simpson index and Coverage index. Beta diversity was assessed by principal component analysis (PCA). Two dissimilarity metrics were used: unweighted UniFrac and weighted UniFrac. Beta diversity analysis implemented in the R phyloseq pack-age. A P-value $<0.05$ was considered to be statistically significant.

\section{Result}


143 Group characteristics

144 A total of 180 participants were included in this study, with an average age of $48.7 \pm 13.4$ years.

145 The distribution of age and sex was similar in the T2DM group, the preDM group and the control 146 group. There were no statistically significant differences in smoking and drinking between the 147 three groups $(P>0.05$, Table 1). Analysis of the participants'FFQs showed

148 significant differences in the intake frequency of cereal grains, mutton, eggs and products, 149 potatoes and sweet potatoes, milk, and yogurt among participants in the three groups $(P<0.05)$. $150(P<0.05$, Table 2$)$. here was no significant difference in the frequency of intake of rice, flour, 151 meat, fowl, fruits and vegetables among the three study groups.

152

\section{Composition and diversity of the gut microbiome} respectively (Figure 2a, 2b).

In our study, the Observed species, Chaol and ACE indexes were used to evaluate the richness of the microbiota, and the Shannon index, Simpson index and Coverage index were used to evaluate the microbiota diversity. The results of the alpha diversity analysis showed that there were no significant differences among the three groups, including ACE, Chao1, Coverage, Observed, Shannon and Simpson indexes (Figure 1). The bacterial community composition, assessed by principal coordinate analysis (PCoA) based on unweighted UniFrac and weighted UniFrac distances, indicated that individuals in the T2DM group and the other two groups clustered separately, presenting $31.75 \%$ and $24.2 \%$ of the total variance on the $\mathrm{x}$-axis and $\mathrm{y}$-axis,

Among the 180 samples tested, there were 366 different bacterial species, 217 different genera, 85 families, 27 classes and 19 phyla. The five most abundant phyla identified were Bacteroidetes, Firmicutes, Proteobacteria, Actinomycetes, and Fusobacteria (Figure 3). The relative abundance of Bacteroidetes and Firmicutes was $43.3 \%$ and $45.1 \%$, respectively, in the T2DM group, $44.9 \%$ and $41.3 \%$ in the preDM group, and $44.5 \%$ and $44.7 \%$ in the control group. Differences in relative abundance among the three groups are presented in Table 3. Compared with the control group, the abundance of phylum Proteobacteria was significantly higher in the preDM group $(P=0.006)$. Proteobacteria were also more abundant in the T2DM group compared 
171

172

173

174

175

176

177

178

179

180

181

182

183

184

185

186

187

188

189

190

191

192

193

194

195

196

197

with the control, though the difference was not significant $(P>0.05)$. Except for Proteobacteria, no statistically significant differences were found among the three groups at the phylum level. At the class level, only class Negativicutes in the T2DM group was more abundant when compared with the other two groups, among the 27 classes (respectively $P=0.017, P=004$ ). Ten genera out of 217 were identified to have differences among the three groups. Compared with the control group, the relative abundance of Prevotella and Alloprevotella was significantly higher in the T2DM group ( $P=0.016, P=0.018$ ), while genus Paraprevotella from phylum Bacteroidetes was less abundant in the T2DM group and preDM group than in the control group $(P=0.011$, $P=0.045)$. Bacteroides was found to be significantly lower in the T2DM compared with the preDM and control groups $(P=0.019, P=0.002)$. Megasphaera was more abundant in the T2DM and preDM groups compared with control $(P=004, P=0.038)$.

182

\section{Discussion}

A total of 180 participants ( 60 healthy, 60 preDM and $60 \mathrm{~T} 2 \mathrm{DM}$ ) were recruited in this study. We evaluated the diversity and compositional changes in the gut microbiota of healthy, preDM and T2DM participants. The five most abundant phyla identified were: Bacteroides, Firmicutes, Proteobacteria, Actinomycetes, and Fusobacteria, which was consistent with previous research(Hollister et al. 2014). Type 2 diabetes may be associated with changes in the balance of gut microbiota, but not with simple changes in the role or diversity of single microbes. Wu et al.(Wu et al. 2010) compared the bacterial diversity of patients with T2DM and non-diabetic individuals, and found that there was no significant difference in bacterial diversity between the two groups, but they noticed a remarkable difference in the numbers of a few bacterial phyla, genera and species. Qin et al. conducted a study on 345 Chinese people and found no difference in microbial diversity between non-diabetic individuals and patients with T2DM; however, differences were found in composition and function, including butyrate-producing bacteria, opportunistic pathogens, and species that may reduce sulfate and degrade mucin(Qin et al. 2012). Our study did not find any difference between T2DM and preDM and the control group in the 
198

199

200

201

202

203

204

205

206

207

208

209

210

211

212

213

214

215

216

217

218

219

220

221

222

223

224

diversity of the gut microbiome but, when compared with the control group, both T2DM and preDM groups had imbalance of the gut microbiome, and changes at the level of genus and class.

The complex interactions between the gut microbiome and gut mucosa may play a key role in the pathogenesis of T2DM, similar to obesity, inflammatory bowel disease and other diseases(Bamola et al. 2017); this may be related to an imbalance of the microbiome that may affect metabolism and cause inflammation. Larsen et al.(Larsen et al. 2010a) found that the gut microbiome of patients with T2DM and non-diabetic individuals showed significant differences in the distribution characteristics of Lactobacillus, Bacteroides, Clostridium, Bifidobacterium and Proteobacteria. In patients with T2DM, Bifidobacteria, Bacteroidetes, and Firmicutes (Lactobacillus) were significantly lower than in the non-diabetic group, but the proportion of Proteobacteria was higher; the reason may be that, in T2DM, glucose metabolic abnormalities and increased glucose metabolites cause an increase in the numbers of pathogenic bacteria in the intestine, thus causing inflammation and insulin resistance. In contrast, however, Mansour Sedighi et al.(Sedighi et al. 2017) found that Lactobacillus was significantly more common in patients with T2DM than in healthy controls, and Bifidobacteria were significantly lower in T2DM. In our study, compared with the healthy control group, the proportion of Lactobacillus was higher in T2DM, but decreased in preDM; the proportion of bifidobacteria was lower in T2DM and preDM, but no significant difference was found.

In this study, we did not find any difference in Firmicutes and Bacteroidetes among the T2DM, preDM and control groups, as also reported by Lambeth et al.(Lambeth et al. 2015). Firmicutes are associated with fat digestion and their increased abundance is known to be associated with obesity. Bacteroidetes play a key role in the production of short-chain fatty acids (SCFAs). It is also believed that Firmicutes and Bacteroidetes can enhance the absorption of monosaccharides in the gut, thus increasing the production of hepatic triglycerides and leading to insulin resistance(Qin et al. 2012; Zhang et al. 2013). However, the results for Firmicutes and Bacteroidetes in diabetic patients differ. Aftab Ahmad et al.(Ahmad et al. 2019b) found a high proportion of Firmicutes and a reduced number of Bacteroidetes in obese patients with T2DM; 
225

226

227

228

229

230

231

232

233

234

235

236

237

238

239

240

241

242

243

244

245

246

247

248

249

250

251

Firmicutes were enriched in T2DM and Bacteroidetes were found in lower numbers, resulting in a high ratio of Firmicutes and Bacteroidetes(Navab-Moghadam et al. 2017; Zhang et al. 2013); however, some other findings are contrary to this(Larsen et al. 2010b; Palacios et al. 2017). Some studies have also demonstrated a significant difference in the Firmicutes/BacTeroiDetes ratio between thin and obese individuals(Heinsen et al. 2016).

We found that Proteobacteria and Escherichia/Shigella were more common in patients with preDM compared with control, and also higher in patients with T2DM, but not significantly. The outer membrane of these bacteria contains lipopolysaccharide (LPS), which is a cellular membrane component of gram-negative bacteria and is increased in both obesity and in patients with T2DM(Sun et al. 2010). LPS can cause metabolic endotoxemia, which is associated with oxidative stress, macrophage secreted elements and inflammatory markers that induce insulin resistance(Momin et al. 2016). Previous findings have indicated that the gut microbiome of patients with T2DM is relatively rich in Gram-negative bacteria when compared with healthy individuals, especially Proteobacteria and Bacteroidetes(Larsen et al. 2010b). In this study, we found a significantly higher abundance of the gram-negative bacterium Haemophilus in patients with preDM compared with healthy individuals. We also found that the T2DM group had higher levels of Prevotella, similar to that found in previous research(Ahmad et al. 2019b; Sedighi et al. 2017; Zhang et al. 2013). This species is related to elevated levels of proinflammatory cytokines, low grade inflammation, and insulin resistance(Leite et al. 2017). In 2016, Copenhagen University and the Danish University of Science and Technology found that serum levels of branched chain amino acids (BCAAs) were increased in diabetic patients, among 277 healthy people without diabetes and 75 patients with T2DM. Prevotella copri and Bacteroides vulgatus were identified as the main species driving the association between biosynthesis of BCAAs and insulin resistance; it was found that Prevotella copri can induce insulin resistance, aggravate glucose intolerance and augment circulating levels of BCAAs in mice fed Prevotella bacteria after 3 weeks(Pedersen et al. 2016). However, the role of Prevotella in human gut microbiome is controversial. It was also recognized as positively associated with the production of health- 
252 promoting compounds such as short-chain fatty acids, an improved glucose metabolism or an

253

254

255

256

257

258

259

260

261

262

263

264

265

266

267

268

269

270

271

272

273

274

275

276

277

278

overall anti-inflammatory effect(De Vadder et al. 2016; Kovatcheva-Datchary et al. 2015). A

recent study of Italians with different dietary habits found that Prevotella's effect on diabetes was related to dietary factors and different strains(De Filippis et al. 2019). In addition, our study found that family Negativicutes, belonging to phylum Firmicutes, and Megasphaera were increased in both the preDM and T2DM groups; the genera Bacteroides and Paraprevotella were reduced in patients with T2DM. Above we discussed the similarities and differences in microbial characteristics associated with T2DM and preDM, and we compared our results with those of different previous studies, as shown in Table S1. Controversial results regarding the characteristics of gut microbiome in patients with T2DM may be related to various factors, such as ethnicity, genetics, environment, geographic and climatic conditions, possible underlying diseases, lifestyle, and dietary habits of study subjects. For example, people living in different regions may have different intestinal microbial structures due to long-term differences in their dietary patterns. Melanie et al.(Deschasaux et al. 2018) found Moroccans, Turks and Ghanaians had a predominance of Prevotella in their intestines and Surinamese in Africa and South Asia had a predominance of Bacteroides; The subjects in this study and previous studies were located in different regions, and their dietary patterns may be quite different. For another example, differences in study methods (case control matching factors, diabetes diagnosis time, PCR specific primers and probe design, etc.) may also lead to controversial results in gut microbiome characteristics in T2DM patients. Therefore, more researches are still needed to elucidate the correct processes of gut microbiome changes associated with T2DM disease progression as well as lifestyle changes, and to gain a more detailed understanding of the role of gut microbiome composition in disease status.

There are some potential confounding factors to note when assessing gut microbiota, although attempts were made to minimize confounding variables, as much as possible, by selecting healthy controls and patients of similar age groups and sex. However, first, we lacked indicators such as height and weight to calculate participants' body mass index (BMI): the known 
279

280

281

282

283

284

285

286

287

288

289

290

291

292

293

294

295

296

297

298

299

300

301

302

303

304

305

association between BMI, obesity and gut microbiome could have affected the results(Ahmad et al. 2019b; Le Chatelier et al. 2013). Diabetics are often associated with obesity, which is related to high abundance of Firmicutes and low abundance of Bacteroides, the diversity of gut microbiome in obese patients was significantly lower than that in normal population(Ahmad et al. 2019a). when obese people diet and lose weight, the proportion of Bacteroidetes/ Firmicutes will increase(Ridaura et al. 2013). A German study(Thingholm et al. 2019) showed that alphadiversity of gut microbiome was significantly reduced in obese subjects (compared to lean healthy subjects), while there was no significant difference between obese subjects and obese T2DM. Comparing obese individuals with and without T2D showed only modest associations between the microbiome and T2D once medication and diet were also factored out, mostly characterized by a nominal increased abundance of Escherichia/Shigella. In this study, although the differences of alpha-diversity between diabetes and normal people and of Firmicutes and Escherichia/Shigella among the three groups were not detected, in the microbiome in which differences were detected, for example, Bacteroides was significantly reduced in diabetic group, possibly due to unknown BMI confounding. Second, Diet is a known factor affecting the development of the human intestinal microbiota. High intakes of carbohydrate, fat and protein are associated with increases in Clostridium IV and XI and decreases in the genera Bifidobacterium and Lactobacillus(Yamaguchi et al. 2016). In addition, studies have shown that, compared with a low dietary fiber group, the abundance of Bifidobacterium and Lactobacillus was higher in a group consuming high dietary fiber(So et al. 2018). Through the dietary FFQ survey, we compared differences in the frequency of dietary intake and found that, except for a few foods, the intake frequency of most foods was not significantly different in the three groups, especially rice, meat, vegetables and fruits, which have a greater influence on the intestinal microbiome. Therefore, to a certain extent, the participants included had similar dietary habits, and this also partly reduced the influence of diet on our results. Thirdly, metformin and other drugs have been associated with changes in the gut microbiome, and studies have found that there was an increase in Firmicutes and decrease in Bacteroidetes in patients taking 
306

307

308

309

310

311

312

313

314

315

316

317

318

319

320

321

322

323

324

325

326

327

328

329

330

331

332

metformin(Forslund et al. 2015; Napolitano et al. 2014). So we included pre-diabetes and newly diagnosed diabetes, both of which generally do not use antihyperglycemic drugs, in order to reduce the impact of such drugs use in our study, and it can't deny that the lack of a medication history of the study subjects still does not completely exclude their non-use of drugs. Finally, Prediabetes are highly various groups, including impaired fasting glucose(IFG) and impaired glucose tolerance(IGT), therefore putting all preDM in one subgroup to analyse may not detect true differences in the gut microbiome profiles.

\section{Conclusions}

In conclusion, this study reported changes in the gut microbiome associated with both preDM and T2DM, especially at the genus level. By studying the relationship between diversity and composition of the gut microbiome and metabolic diseases (such as T2DM), earlier intervention is possible to restore the microbiome to the normal state. PreDM may have an impact on the intestinal microflora in transition to T2DM, which may be altered through changes in lifestyle factors, including dietary habits and physical activity, weight management, and the use of appropriate probiotics and other substances that have a substantial impact on the gut microbiome.

\section{Acknowledgements}

Author Contributions: T.T. and D.J. conceived and designed the study. Material preparation and data collection were performed by T. T., C. Z.; Z. Z., L.L. and L.T. analysed and interpreted the data. The original draft of the manuscript was written by Z.Z. and D.J. and T.T. reviewed and edited the manuscript. Project administration, D.J.

\section{References}

Ahmad A, Yang W, Chen G, Shafiq M, Javed S, Ali Zaidi S, Shahid R, Liu C, and Bokhari H. 2019a. Analysis of gut microbiota of obese individuals with type 2 diabetes and healthy individuals. PloS one 14:e0226372. 10.1371/journal.pone. 0226372

Peer) reviewing PDF | (2020:08:52130:2:0:NEW 7 Jan 2021) 
333

334

335

336

337

338

339

340

341

342

343

344

345

346

347

348

349

350

351

352

353

354

355

356

357

358

359

360

361

362

363

364

365

366

367

368

369

370

371

372

373

Ahmad A, Yang W, Chen G, Shafiq M, Javed S, Ali Zaidi SS, Shahid R, Liu C, and Bokhari H. 2019b. Analysis of gut microbiota of obese individuals with type 2 diabetes and healthy individuals. PLoS One 14:e0226372. 10.1371/journal.pone.0226372

Allin KH, Tremaroli V, Caesar R, Jensen BAH, Damgaard MTF, Bahl MI, Licht TR, Hansen TH, Nielsen T, Dantoft TM, Linneberg A, Jorgensen T, Vestergaard H, Kristiansen K, Franks PW, consortium I-D, Hansen T, Backhed F, and Pedersen O. 2018. Aberrant intestinal microbiota in individuals with prediabetes. Diabetologia 61:810820. 10.1007/s00125-018-4550-1

Bamola VD, Ghosh A, Kapardar RK, Lal B, Cheema S, Sarma P, and Chaudhry R. 2017. Gut microbial diversity in health and disease: experience of healthy Indian subjects, and colon carcinoma and inflammatory bowel disease patients. Microb Ecol Health Dis 28:1322447. 10.1080/16512235.2017.1322447

Cho NH, Shaw JE, Karuranga S, Huang Y, da Rocha Fernandes JD, Ohlrogge AW, and Malanda B. 2018. IDF Diabetes Atlas: Global estimates of diabetes prevalence for 2017 and projections for 2045. Diabetes Res Clin Pract 138:271-281. 10.1016/j.diabres.2018.02.023

De Filippis F, Pasolli E, Tett A, Tarallo S, Naccarati A, De Angelis M, Neviani E, Cocolin L, Gobbetti M, Segata N, and Ercolini D. 2019. Distinct Genetic and Functional Traits of Human Intestinal Prevotella copri Strains Are Associated with Different Habitual Diets. Cell host \& microbe 25:444-453.e443. 10.1016/j.chom.2019.01.004

De Vadder F, Kovatcheva-Datchary P, Zitoun C, Duchampt A, Bäckhed F, and Mithieux G. 2016. Microbiota-Produced Succinate Improves Glucose Homeostasis via Intestinal Gluconeogenesis. Cell metabolism 24:151-157. 10.1016/j.cmet.2016.06.013

Deschasaux M, Bouter K, Prodan A, Levin E, Groen A, Herrema H, Tremaroli V, Bakker G, Attaye I, Pinto-Sietsma S, van Raalte D, Snijder M, Nicolaou M, Peters R, Zwinderman A, Bäckhed F, and Nieuwdorp M. 2018. Depicting the composition of gut microbiota in a population with varied ethnic origins but shared geography. Nature medicine 24:1526-1531. 10.1038/s41591-018-0160-1

Festi D, Schiumerini R, Eusebi LH, Marasco G, Taddia M, and Colecchia A. 2014. Gut microbiota and metabolic syndrome. World J Gastroenterol 20:16079-16094. 10.3748/wjg.v20.i43.16079

Forslund K, Hildebrand F, Nielsen T, Falony G, Le Chatelier E, Sunagawa S, Prifti E, Vieira-Silva S, Gudmundsdottir V, Pedersen HK, Arumugam M, Kristiansen K, Voigt AY, Vestergaard H, Hercog R, Costea PI, Kultima JR, Li J, Jorgensen T, Levenez F, Dore J, Meta HITc, Nielsen HB, Brunak S, Raes J, Hansen T, Wang J, Ehrlich SD, Bork $P$, and Pedersen O. 2015. Disentangling type 2 diabetes and metformin treatment signatures in the human gut microbiota. Nature 528:262-266. 10.1038/nature15766

Gaike AH, Paul D, Bhute S, Dhotre DP, Pande P, Upadhyaya S, Reddy Y, Sampath R, Ghosh D, Chandraprabha D, Acharya J, Banerjee G, Sinkar VP, Ghaskadbi SS, and Shouche YS. 2020. The Gut Microbial Diversity of Newly Diagnosed Diabetics but Not of Prediabetics Is Significantly Different from That of Healthy Nondiabetics. mSystems 5. 10.1128/mSystems.00578-19

Heinsen FA, Fangmann D, Muller N, Schulte DM, Ruhlemann MC, Turk K, Settgast U, Lieb W, Baines JF, Schreiber S, Franke A, and Laudes M. 2016. Beneficial Effects of a Dietary Weight Loss Intervention on Human Gut Microbiome Diversity and Metabolism Are Not Sustained during Weight Maintenance. Obes Facts 9:379391. 10.1159/000449506

Hollister EB, Gao C, and Versalovic J. 2014. Compositional and functional features of the gastrointestinal microbiome and their effects on human health. Gastroenterology 146:1449-1458. 10.1053/j.gastro.2014.01.052

Peer) reviewing PDF | (2020:08:52130:2:0:NEW 7 Jan 2021) 
374

375

376

377

378

379

380

381

382

383

384

385

386

387

388

389

390

391

392

393

394

395

396

397

398

399

400

401

402

403

404

405

406

407

408

409

410

411

412

413

414
Karlsson FH, Tremaroli V, Nookaew I, Bergstrom G, Behre CJ, Fagerberg B, Nielsen J, and Backhed F. 2013. Gut metagenome in European women with normal, impaired and diabetic glucose control. Nature 498:99-103. $10.1038 /$ nature12198

Komaroff AL. 2017. The Microbiome and Risk for Obesity and Diabetes. JAMA 317:355-356. 10.1001/jama.2016.20099

Kovatcheva-Datchary P, Nilsson A, Akrami R, Lee Y, De Vadder F, Arora T, Hallen A, Martens E, Björck I, and Bäckhed F. 2015. Dietary Fiber-Induced Improvement in Glucose Metabolism Is Associated with Increased Abundance of Prevotella. Cell metabolism 22:971-982. 10.1016/j.cmet.2015.10.001

Lambeth SM, Carson T, Lowe J, Ramaraj T, Leff JW, Luo L, Bell CJ, and Shah VO. 2015. Composition, Diversity and Abundance of Gut Microbiome in Prediabetes and Type 2 Diabetes. J Diabetes Obes 2:1-7. 10.15436/23760949.15.031

Larsen N, Vogensen F, van den Berg F, Nielsen D, Andreasen A, Pedersen B, Al-Soud W, Sørensen S, Hansen L, and Jakobsen M. 2010a. Gut microbiota in human adults with type 2 diabetes differs from non-diabetic adults. PloS one 5:e9085. 10.1371/journal.pone.0009085

Larsen N, Vogensen FK, van den Berg FW, Nielsen DS, Andreasen AS, Pedersen BK, Al-Soud WA, Sorensen SJ, Hansen LH, and Jakobsen M. 2010b. Gut microbiota in human adults with type 2 diabetes differs from non-diabetic adults. PLoS One 5:e9085. 10.1371/journal.pone.0009085

Le Chatelier E, Nielsen T, Qin J, Prifti E, Hildebrand F, Falony G, Almeida M, Arumugam M, Batto JM, Kennedy S, Leonard P, Li J, Burgdorf K, Grarup N, Jorgensen T, Brandslund I, Nielsen HB, Juncker AS, Bertalan M, Levenez F, Pons N, Rasmussen S, Sunagawa S, Tap J, Tims S, Zoetendal EG, Brunak S, Clement K, Dore J, Kleerebezem M, Kristiansen K, Renault P, Sicheritz-Ponten T, de Vos WM, Zucker JD, Raes J, Hansen T, Meta HITc, Bork P, Wang J, Ehrlich SD, and Pedersen O. 2013. Richness of human gut microbiome correlates with metabolic markers. Nature 500:541-546. 10.1038/nature12506

Leite AZ, Rodrigues NC, Gonzaga MI, Paiolo JCC, de Souza CA, Stefanutto NAV, Omori WP, Pinheiro DG, Brisotti JL, Matheucci Junior E, Mariano VS, and de Oliveira GLV. 2017. Detection of Increased Plasma Interleukin-6 Levels and Prevalence of Prevotella copri and Bacteroides vulgatus in the Feces of Type 2 Diabetes Patients. Front Immunol 8:1107. 10.3389/fimmu.2017.01107

Lynch SV, and Pedersen O. 2016. The Human Intestinal Microbiome in Health and Disease. N Engl J Med 375:23692379. 10.1056/NEJMra1600266

Ma Q, Li Y, Li P, Wang M, Wang J, Tang Z, Wang T, Luo L, Wang C, Wang T, and Zhao B. 2019. Research progress in the relationship between type 2 diabetes mellitus and intestinal flora. Biomed Pharmacother 117:109138. 10.1016/j.biopha.2019.109138

Momin AA, Bankar MP, and Bhoite GM. 2016. Study of Common Genetic Variant S447X in Lipoprotein Lipase and Its Association with Lipids and Lipoproteins in Type 2 Diabetic Patients. Indian J Clin Biochem 31:286-293. 10.1007/s12291-015-0531-z

Napolitano A, Miller S, Nicholls AW, Baker D, Van Horn S, Thomas E, Rajpal D, Spivak A, Brown JR, and Nunez DJ. 2014. Novel gut-based pharmacology of metformin in patients with type 2 diabetes mellitus. PLoS One 9:e100778. 10.1371/journal.pone.0100778

Naseer MI, Bibi F, Alqahtani MH, Chaudhary AG, Azhar EI, Kamal MA, and Yasir M. 2014. Role of gut microbiota in obesity, type 2 diabetes and Alzheimer's disease. CNS Neurol Disord Drug Targets 13:305-311. $10.2174 / 18715273113126660147$

Peer) reviewing PDF | (2020:08:52130:2:0:NEW 7 Jan 2021) 
415

416

417

418

419

420

421

422

423

424

425

426

427

428

429

430

431

432

433

434

435

436

437

438

439

440

441

442

443

444

445

446

447

448

449

450

451

452

453

454

455

Navab-Moghadam F, Sedighi M, Khamseh ME, Alaei-Shahmiri F, Talebi M, Razavi S, and Amirmozafari N. 2017. The association of type II diabetes with gut microbiota composition. Microb Pathog 110:630-636. 10.1016/j.micpath.2017.07.034

Palacios T, Vitetta L, Coulson S, Madigan CD, Denyer GS, and Caterson ID. 2017. The effect of a novel probiotic on metabolic biomarkers in adults with prediabetes and recently diagnosed type 2 diabetes mellitus: study protocol for a randomized controlled trial. Trials 18:7. 10.1186/s13063-016-1762-x

Pedersen HK, Gudmundsdottir V, Nielsen HB, Hyotylainen T, Nielsen T, Jensen BA, Forslund K, Hildebrand F, Prifti E, Falony G, Le Chatelier E, Levenez F, Dore J, Mattila I, Plichta DR, Poho P, Hellgren LI, Arumugam M, Sunagawa S, Vieira-Silva S, Jorgensen T, Holm JB, Trost K, Meta HITC, Kristiansen K, Brix S, Raes J, Wang J, Hansen T, Bork P, Brunak S, Oresic M, Ehrlich SD, and Pedersen O. 2016. Human gut microbes impact host serum metabolome and insulin sensitivity. Nature 535:376-381. 10.1038/nature18646

Qin J, Li Y, Cai Z, Li S, Zhu J, Zhang F, Liang S, Zhang W, Guan Y, Shen D, Peng Y, Zhang D, Jie Z, Wu W, Qin Y, Xue W, Li J, Han L, Lu D, Wu P, Dai Y, Sun X, Li Z, Tang A, Zhong S, Li X, Chen W, Xu R, Wang M, Feng Q, Gong M, Yu J, Zhang Y, Zhang M, Hansen T, Sanchez G, Raes J, Falony G, Okuda S, Almeida M, LeChatelier E, Renault P, Pons N, Batto JM, Zhang Z, Chen H, Yang R, Zheng W, Li S, Yang H, Wang J, Ehrlich SD, Nielsen R, Pedersen $\mathrm{O}$, Kristiansen $\mathrm{K}$, and Wang J. 2012. A metagenome-wide association study of gut microbiota in type 2 diabetes. Nature 490:55-60. 10.1038/nature11450

Qiu J, Zhou H, Jing Y, and Dong C. 2019. Association between blood microbiome and type 2 diabetes mellitus: A nested case-control study. J Clin Lab Anal 33:e22842. 10.1002/jcla.22842

Ridaura V, Faith J, Rey F, Cheng J, Duncan A, Kau A, Griffin N, Lombard V, Henrissat B, Bain J, Muehlbauer M, Ilkayeva O, Semenkovich C, Funai K, Hayashi D, Lyle B, Martini M, Ursell L, Clemente J, Van Treuren W, Walters W, Knight R, Newgard C, Heath A, and Gordon J. 2013. Gut microbiota from twins discordant for obesity modulate metabolism in mice. Science (New York, NY) 341:1241214. 10.1126/science.1241214

Sato J, Kanazawa A, Ikeda F, Yoshihara T, Goto H, Abe H, Komiya K, Kawaguchi M, Shimizu T, Ogihara T, Tamura Y, Sakurai Y, Yamamoto R, Mita T, Fujitani Y, Fukuda H, Nomoto K, Takahashi T, Asahara T, Hirose T, Nagata S, Yamashiro Y, and Watada H. 2014. Gut dysbiosis and detection of "live gut bacteria" in blood of Japanese patients with type 2 diabetes. Diabetes Care 37:2343-2350. 10.2337/dc13-2817

Sedighi M, Razavi S, Navab-Moghadam F, Khamseh ME, Alaei-Shahmiri F, Mehrtash A, and Amirmozafari N. 2017. Comparison of gut microbiota in adult patients with type 2 diabetes and healthy individuals. Microb Pathog 111:362-369. 10.1016/j.micpath.2017.08.038

So D, Whelan K, Rossi M, Morrison M, Holtmann G, Kelly JT, Shanahan ER, Staudacher HM, and Campbell KL. 2018. Dietary fiber intervention on gut microbiota composition in healthy adults: a systematic review and metaanalysis. Am J Clin Nutr 107:965-983. 10.1093/ajen/nqy041

Sun L, Yu Z, Ye X, Zou S, Li H, Yu D, Wu H, Chen Y, Dore J, Clement K, Hu FB, and Lin X. 2010. A marker of endotoxemia is associated with obesity and related metabolic disorders in apparently healthy Chinese. Diabetes Care 33:1925-1932. 10.2337/dc10-0340

Tabak AG, Herder C, Rathmann W, Brunner EJ, and Kivimaki M. 2012. Prediabetes: a high-risk state for diabetes development. Lancet 379:2279-2290. 10.1016/S0140-6736(12)60283-9

Thingholm L, Rühlemann M, Koch M, Fuqua B, Laucke G, Boehm R, Bang C, Franzosa E, Hübenthal M, Rahnavard A, Frost F, Lloyd-Price J, Schirmer M, Lusis A, Vulpe C, Lerch M, Homuth G, Kacprowski T, Schmidt C, Nöthlings U, Karlsen T, Lieb W, Laudes M, Franke A, and Huttenhower C. 2019. Obese Individuals with and without

PeerJ reviewing PDF | (2020:08:52130:2:0:NEW 7 Jan 2021) 
456

457

458

459

460

461

462

463

464

465

466

467

468

469

470

471

472

473

474

475
Type 2 Diabetes Show Different Gut Microbial Functional Capacity and Composition. Cell host \& microbe 26:252-264.e210. 10.1016/j.chom.2019.07.004

Wang L, Gao P, Zhang M, Huang Z, Zhang D, Deng Q, Li Y, Zhao Z, Qin X, Jin D, Zhou M, Tang X, Hu Y, and Wang L. 2017. Prevalence and Ethnic Pattern of Diabetes and Prediabetes in China in 2013. JAMA 317:2515-2523. 10.1001/jama.2017.7596

Wu X, Ma C, Han L, Nawaz M, Gao F, Zhang X, Yu P, Zhao C, Li L, Zhou A, Wang J, Moore JE, Millar BC, and Xu J. 2010. Molecular characterisation of the faecal microbiota in patients with type II diabetes. Curr Microbiol 61:6978. 10.1007/s00284-010-9582-9

Yamaguchi Y, Adachi K, Sugiyama T, Shimozato A, Ebi M, Ogasawara N, Funaki Y, Goto C, Sasaki M, and Kasugai K. 2016. Association of Intestinal Microbiota with Metabolic Markers and Dietary Habits in Patients with Type 2 Diabetes. Digestion 94:66-72. 10.1159/000447690

Zhang X, Shen D, Fang Z, Jie Z, Qiu X, Zhang C, Chen Y, and Ji L. 2013. Human gut microbiota changes reveal the progression of glucose intolerance. PLoS One 8:e71108. 10.1371/journal.pone.0071108

Zhang YJ, Li S, Gan RY, Zhou T, Xu DP, and Li HB. 2015. Impacts of gut bacteria on human health and diseases. Int J Mol Sci 16:7493-7519. 10.3390/ijms16047493

Zhong H, Ren H, Lu Y, Fang C, Hou G, Yang Z, Chen B, Yang F, Zhao Y, Shi Z, Zhou B, Wu J, Zou H, Zi J, Chen J, Bao X, Hu Y, Gao Y, Zhang J, Xu X, Hou Y, Yang H, Wang J, Liu S, Jia H, Madsen L, Brix S, Kristiansen K, Liu F, and Li J. 2019. Distinct gut metagenomics and metaproteomics signatures in prediabetics and treatment-naïve type 2 diabetics. EBioMedicine 47:373-383. 10.1016/j.ebiom.2019.08.048 
Table $\mathbf{1}$ (on next page)

Characteristics of the participants 
1 Table 1 Characteristics of the participants

\begin{tabular}{lllll}
\hline Characteristics & $\mathrm{T} 2 \mathrm{DM}(\mathrm{N}=60)$ & $\operatorname{PreDM}(\mathrm{N}=60)$ & Control(N=60) & $P$ \\
\hline Age(years) & $49.4 \pm 13.2$ & $47.0 \pm 14.1$ & $48.5 \pm 13.3$ & 0.609 \\
Men/Women & $31 / 29$ & $30 / 30$ & $29 / 31$ & 0.860 \\
fasting blood-glucose & $9.93 \pm 3.55$ & $6.43 \pm 0.30$ & $4.79 \pm 0.47$ & 0.001 \\
Smoke & $17(28.3)$ & $17(28.8)$ & $15(25.4)$ & 0.894 \\
Drink & $8(13.3)$ & $11(18.3)$ & $13(21.7)$ & 0.486 \\
\hline
\end{tabular}

2 
Table 2 (on next page)

Dietary frequency questionnaires of study participants 
Table 2 Dietary frequency questionnaires of study participants

\begin{tabular}{|c|c|c|c|c|c|c|c|}
\hline \multirow{3}{*}{ categories } & \multicolumn{6}{|c|}{ Dietary frequency n $(\%)$} & \multirow{3}{*}{$P$} \\
\hline & \multicolumn{2}{|l|}{ control $(\mathrm{N}=60)$} & \multicolumn{2}{|l|}{$\mathrm{T} 2 \mathrm{DM}(\mathrm{N}=60)$} & \multicolumn{2}{|l|}{$\operatorname{PreDM}(\mathrm{N}=60)$} & \\
\hline & $\geq 1 \sim 3$ times/week & little or not & $\geq 1 \sim 3$ times/week & little or not & $\geq 1 \sim 3$ times/week & little or not & \\
\hline Rice & $56(93.3)$ & $4(6.7)$ & $48(80.0)$ & $12(20.0)$ & $54(90.0)$ & $6(10.0)$ & 0.068 \\
\hline Flour & $57(95.0)$ & $3(5.0)$ & $56(93.3)$ & $4(6.7)$ & $57(95.0)$ & $3(5.0)$ & 0.902 \\
\hline $\begin{array}{l}\text { Cereal(corn,sorghum, } \\
\text { millet) }\end{array}$ & $35(58.3)^{b}$ & $25(41.7)$ & $21(35.0)^{\mathrm{a}}$ & $39(65.0)$ & $22(36.7)^{\mathrm{a}, \mathrm{b}}$ & $38(63.3)$ & 0.016 \\
\hline Pork & $39(65.0)$ & $21(35.0)$ & $34(56.7)$ & $26(43.3)$ & $32(53.3)$ & $28(46.7)$ & 0.410 \\
\hline Beef & $45(75.0)$ & $15(25.0)$ & $43(71.7)$ & $17(28.3)$ & $34(56.7)$ & $26(43.3)$ & 0.073 \\
\hline Fowl & $31(51.7)$ & $29(48.3)$ & $23(38.3)$ & $37(61.7)$ & $22(36.7)$ & $38(63.3)$ & 0.190 \\
\hline Seafood & $21(35.6)$ & $38(64.4)$ & $11(18.3)$ & $49(81.7)$ & $14(23.7)$ & $45(76.3)$ & 0.089 \\
\hline Eggs and their product & $48(80.0)^{b}$ & $12(20.0)$ & $34(56.7)^{\mathrm{a}}$ & $26(43.3)$ & $41(68.3)^{\mathrm{a}, \mathrm{b}}$ & $19(31.7)$ & 0.023 \\
\hline Offal & $5(8.3)$ & $55(91.7)$ & $4(6.7)$ & $56(93.3)$ & $2(3.3)$ & $58(96.7)$ & 0.483 \\
\hline Vegetables & $59(98.3)$ & $1(1.7)$ & $58(96.7)$ & $2(3.3)$ & $59(98.3)$ & $1(1.7)$ & 0.786 \\
\hline Fruits & $56(93.3)$ & $4(6.7)$ & $52(86.7)$ & $8(13.3)$ & $53(88.3)$ & $7(11.7)$ & 0.465 \\
\hline $\begin{array}{l}\text { Potatoes and sweet } \\
\text { potatoes }\end{array}$ & $49(81.7)^{b}$ & $11(18.3)$ & $33(42.5)^{\mathrm{a}}$ & $27(45.0)$ & $45(75.0)^{\mathrm{a}, \mathrm{b}}$ & $15(25.0)$ & 0.004 \\
\hline Beans and their product & $38(63.3)$ & $22(36.7)$ & $32(53.3)$ & $28(46.7)$ & $35(58.3)$ & $25(41.7)$ & 0.539 \\
\hline Milk & $41(69.5)^{b}$ & $19(31.7)$ & $26(43.3)^{\mathrm{a}}$ & $34(56.7)$ & $26(43.3)^{\mathrm{a}}$ & $34(56.7)$ & 0.007 \\
\hline Yogurt (solid, liquid) & $36(60.0)^{b}$ & $24(40.0)$ & $16(26.7)^{\mathrm{a}}$ & $44(73.3)$ & $14(23.3)^{\mathrm{a}}$ & $46(76.7)$ & 0.001 \\
\hline Butter tea & $1(1.7)$ & $59(98.3)$ & $1(1.7)$ & $59(98.3)$ & $2(3.3)$ & $58(96.7)$ & 0.786 \\
\hline Milky tea & $14(23.3)$ & $46(76.7)$ & $10(16.7)$ & $50(83.3)$ & $8(13.3)$ & $52(86.7)$ & 0.345 \\
\hline
\end{tabular}

2 a,b denotes comparison between subgroups at the 0.05 level after adjustment of $p$-values (Bonferroni method) 


\section{Table $\mathbf{3}$ (on next page)}

Relative abundance at phylum, class and genus levels in T2DM, preDM and control groups 
1 Table 3 Relative abundance at phylum, class and genus levels in T2DM, preDM and control 2 groups

\begin{tabular}{|c|c|c|c|c|c|c|c|c|}
\hline \multirow{2}{*}{ Category } & \multirow{2}{*}{ Level } & \multicolumn{3}{|c|}{ Relative abundance(\%) } & \multicolumn{4}{|c|}{$P$ value/FDR $P$ value } \\
\hline & & ND & $\mathrm{DM}$ & PDM & ALL & N vs DM & $\mathrm{N}$ vs PDM & DM vs PDM \\
\hline Firmicutes & Phylum & 44.7 & 45.1 & 41.3 & 0.87 & $0.8781 / 1$ & $0.2098 / 0.671$ & $0.1239 / 0.995$ \\
\hline Negativicutes & Class & 3.37 & 6.50 & 4.01 & 0.0019 & 0.0040 & $0.4635 / 0.833$ & $0.0170 / 0.229$ \\
\hline Finegoldia & genus & 0.0004 & 0.002 & 0.0005 & 0.0084 & $0.0001 / 0.000$ & $0.3592 / 0.676$ & $0.0050 / 0.075$ \\
\hline Megasphaera & genus & 0.06 & 0.58 & 0.39 & 0.0211 & $0.0040 / 0.039$ & $0.0380 / 0.181$ & $0.5114 / 0.892$ \\
\hline Lachnospira & genus & 0.55 & 0.48 & 0.21 & 0.6564 & $0.6534 / 1$ & $0.0120 / 0.072$ & $0.0949 / 0.499$ \\
\hline Lactobacillus & genus & 0.60 & 1.10 & 0.38 & 0.175 & $0.2637 / 0.585$ & $0.5345 / 0.823$ & $0.0809 / 0.463$ \\
\hline Bacteroidetes & Phylum & 44.5 & 43.3 & 44.9 & 0.67 & $0.6883 / 1$ & $0.9060 / 0.959$ & $0.5764 / 0.996$ \\
\hline Bacteroides & genus & 32.0 & 22.1 & 29.7 & 0.0014 & $0.0020 / 0.027$ & $0.4345 / 0.761$ & $0.0190 / 0.175$ \\
\hline Paraprevotella & genus & 0.49 & 0.12 & 0.17 & 0.0075 & $0.0110 / 0.077$ & $0.0450 / 0.192$ & $0.4495 / 0.874$ \\
\hline Prevotella & genus & 7.1 & 15.2 & 9.5 & 0.0109 & $0.0160 / 0.102$ & $0.3856 / 0.690$ & $0.0979 / 0.499$ \\
\hline Alloprevotella & genus & 0.12 & 1.06 & 0.26 & 0.0247 & $0.0180 / 0.111$ & $0.5124 / 0.816$ & $0.0949 / 0.499$ \\
\hline Proteobacteria & Phylum & 5.8 & 7.8 & 10.5 & 0.260 & $0.1678 / 1$ & $0.0060 / 0.048$ & $0.2137 / 0.995$ \\
\hline Helicobacter & genus & 0.0003 & 0.00004 & 0.00004 & 0.0382 & $0.0063 / 0.054$ & $0.0156 / 0.089$ & - \\
\hline Escherichia/Shigella & genus & 1.93 & 2.65 & 4.24 & 0.4848 & $0.3436 / 0.697$ & $0.0290 / 0.141$ & $0.2027 / 0.657$ \\
\hline Haemophilus & genus & 0.11 & 0.52 & 0.48 & 0.1135 & $0.0769 / 0.294$ & $0.0120 / 0.072$ & $0.9240 / 1$ \\
\hline Fusobacteria & Phylum & 0.64 & 0.58 & 0.25 & 0.8824 & $0.9500 / 1$ & $0.4905 / 0.831$ & $0.3047 / 0.995$ \\
\hline Fusobacterium & genus & 0.33 & 0.54 & 0.25 & 0.5391 & $0.5514 / 0.879$ & $0.9730 / 1$ & $0.4106 / 0.861$ \\
\hline Verrucomicrobia & Phylum & 0.38 & 0.37 & 0.38 & 0.98 & $0.9970 / 1$ & $0.9590 / 0.959$ & $0.9550 /-$ \\
\hline Actinobacteria & Phylum & 3.6 & 2.6 & 2.5 & 0.25 & $0.3016 / 1$ & $0.2557 / 0.682$ & $0.9630 /-$ \\
\hline
\end{tabular}


$\begin{array}{lllllllll}\text { Bifidobacterium } & \text { genus } & 3.1 & 1.9 & 1.9 & 0.1260 & 0.1588 / 0.487 & 0.1878 / 0.464 & 0.9690 / 1\end{array}$

3 
Figure 1

Alpha diversity index of the T2DM group, PreDM group and non-diabetes group 

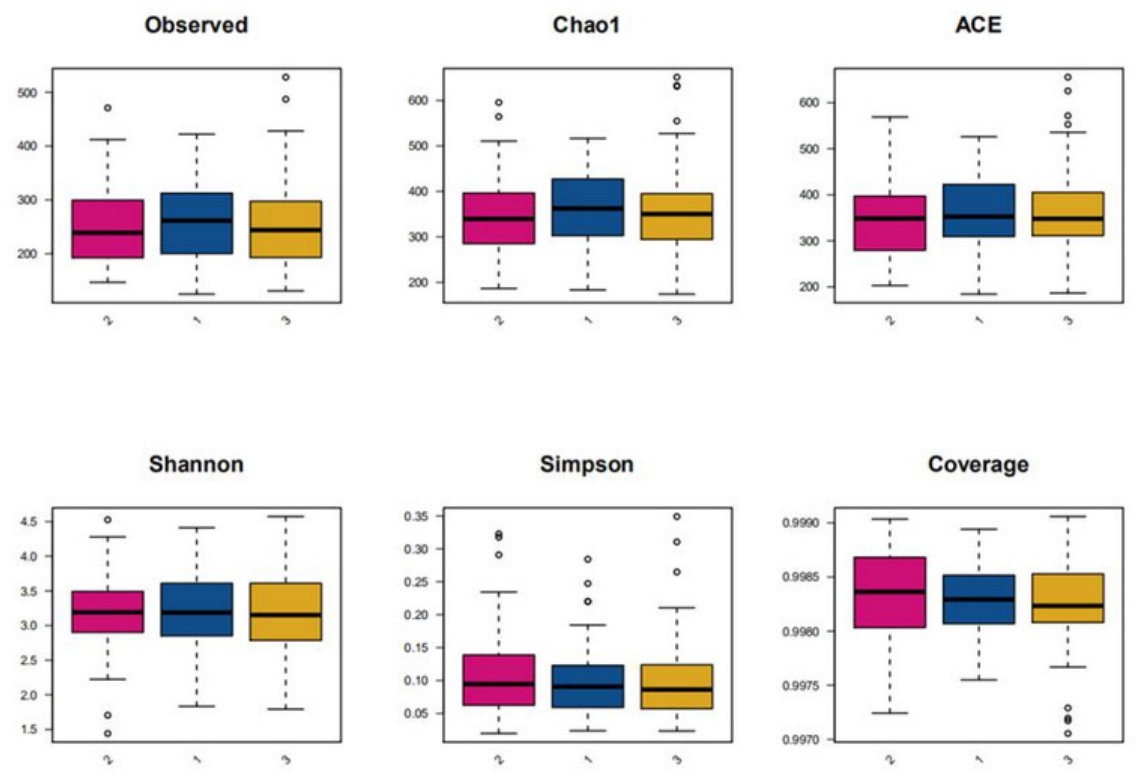

Figure 1. Alpha diversity index of the T2DM group, PreDM group and non-diabetes group (1 for T2DM group, 2 for PreDM group and 3 for control group). 
Figure 2 (on next page)

PCoA of T2DM group, PreDM group and non-diabetes group 


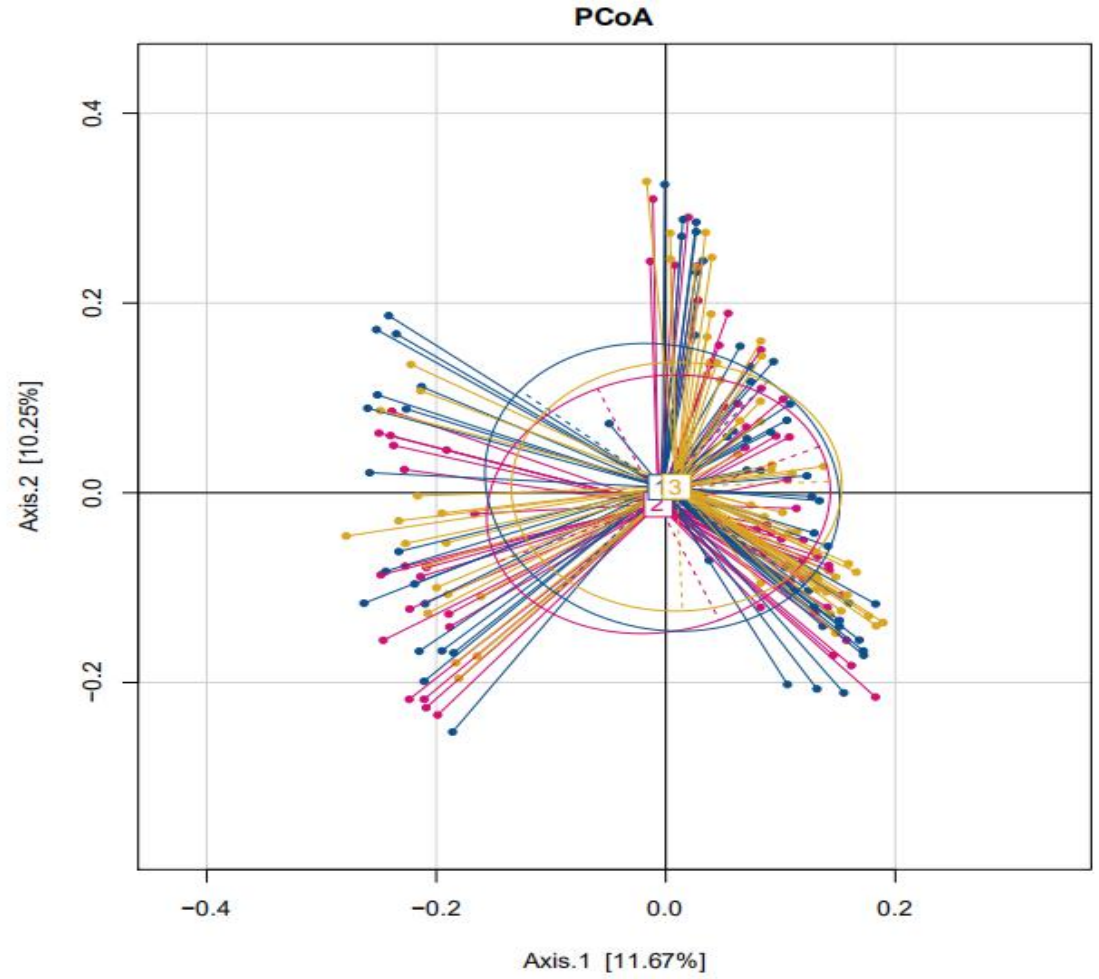

Figure 2.a Unweighted-UniFrac PCoA of T2DM group, PreDM group and non-diabetes group (1 for T2DM group, 2 for PreDM group and 3 for control group).

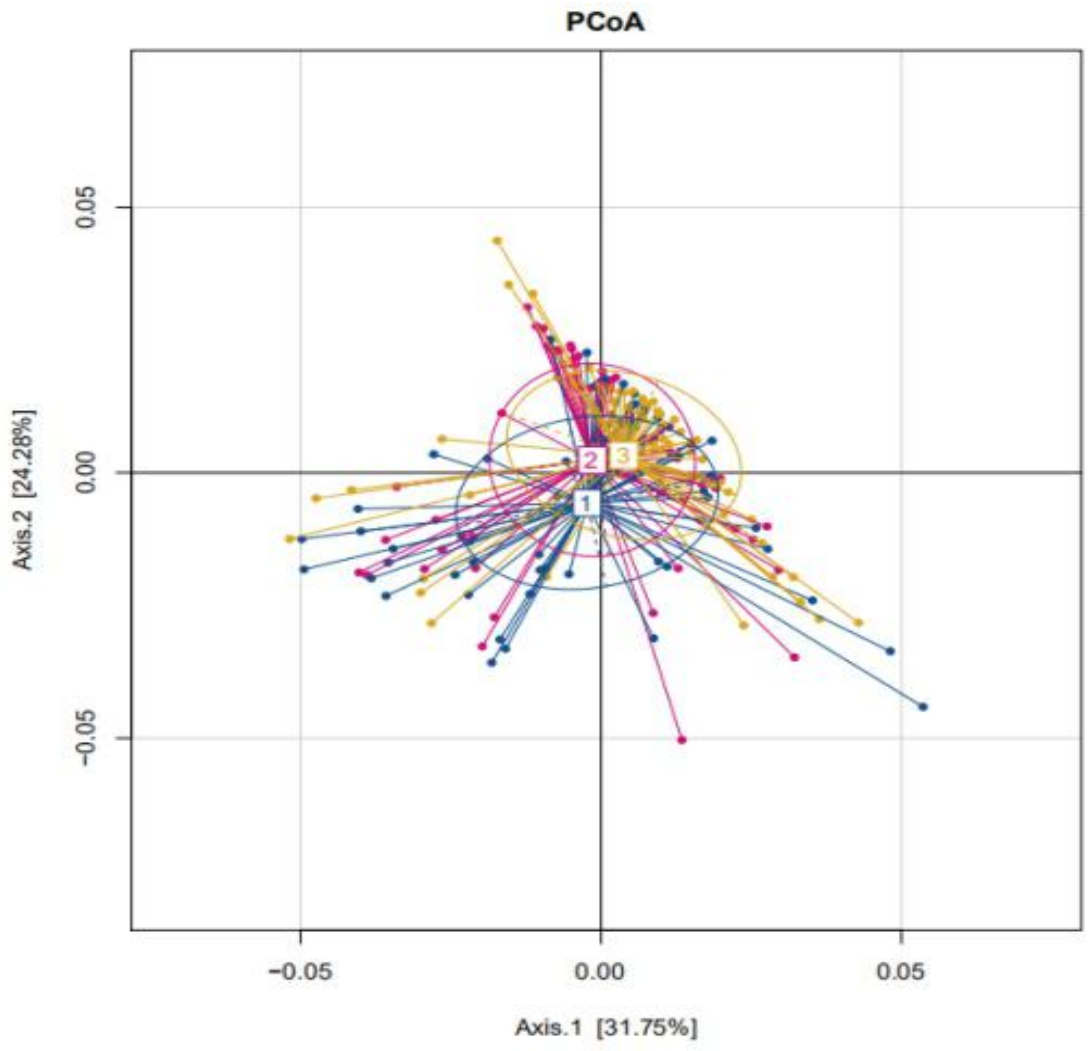

Figure 2. b Weighted-UniFrac PCoA of T2DM group, PreDM group and non-diabetes group. (1 for T2DM group, 2 for PreDM group and 3 for control group). 
Figure 3

Relative richness (gate level) in T2DM group, PreDM group and non-diabetes group 

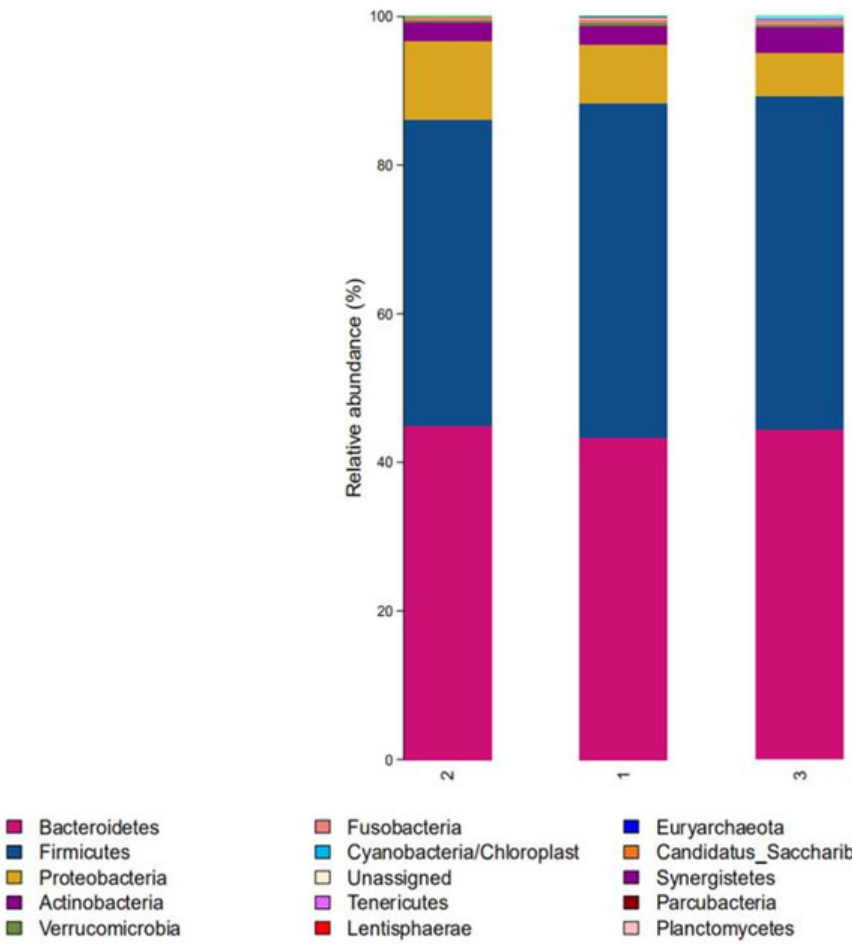

- Euryarchaeota m

Verrucomicrobia

Figure 3. Relative richness (gate level) in T2DM group, PreDM group and non-diabetes group. ( 1 for Type 2 diabetes group, 2 for prediabetes group and 3 for control group). 